\title{
Prevalencia de agrandamiento y retracción gingival en pacientes con tratamiento de ortodoncia
}

\author{
Ana Gabriela Rodríguez Vásquez ${ }^{1}$ \\ Lourdes Karina Fernández García ${ }^{2}$ \\ Edita Hortencia Valladares Trochez ${ }^{3}$
}

\section{RESUMEN}

La clave para el éxito en los tratamientos de ortodoncia van enfocados a un buen diagnóstico, y una excelente comunicación multi e interdisciplinaria con las demás especialidades odontológicas, siempre considerando la prevención como área primordial. El objetivo de este estudio es determinar la prevalencia del agrandamiento y retracción gingival en pacientes con tratamiento de ortodoncia, con el fin de conocer el tipo de alteraciones periodontales prevalentes en pacientes con aparatología ortodóntica.

La muestra utilizada es de 200 pacientes que asisten a atención ortodóntica en el Hospital Odontológico Monseñor Agustín Hombach de la Universidad Católica de Honduras; dicha muestra se calculó en base a un universo de 450 personas con un $5 \%$ de error muestra constituyéndose esta como muestra aleatoria simple. El instrumento de recolección de datos comprendió: una anamnesis (interrogatorios llenados por el paciente) y la parte de historia clínica (llenada por el investigador) en la cual se observó y midió clínicamente el agrandamiento, retracción gingival y placa bacteriana. Los resultados establecidos en el estudio demostraron que la prevalencia del agrandamiento fue de 110 (55\%) pacientes y retracción gingival 21(10\%) pacientes, la deficiencia de higiene bucal es prevalente en pacientes con tratamiento de ortodoncia a causa de la aparatología fija que utilizan, los factores de riesgo (dieta, enfermedades sistémicas medicamentos) no agravan el agrandamiento y retracción gingival en dichos pacientes. En el análisis estadístico de correlación utilizado, se comprobaron las hipótesis de investigación que establecen: existe correlación entre el tiempo del tratamiento de ortodoncia y el agrandamiento gingival en pacientes con trata-

\footnotetext{
${ }^{1}$ Estudiante, Carrera de Cirugía Dental de la Universidad Católica de Honduras "Nuestra Señora Reina de la Paz". anarv.193@gmail.com

${ }^{2}$ Asesor metodológico, Directora del Hospital Odontológico Monseñor Agustín Hombach de la Universidad Católica de Honduras "Nuestra Señora Reina de la Paz"

${ }^{3}$ Asesor técnico, Profesora de Postgrado de Ortodoncia y Ortopedia Maxilofacial de la facultad de Cirugía Dental de la Universidad Católica de Honduras "Nuestra Señora Reina de la Paz"
} 
miento de ortodoncia, en la cual hubo una correlación positiva con un índice de significancia de 0.021, y existe correlación entre la edad del paciente y la retracción gingival con un índice de significancia de 0.011 , que indica una correlación positiva.

Palabras clave: agrandamiento gingival, retracción gingival, tratamiento de ortodoncia, deficiencia de higiene bucal.

\section{ABSTRACT}

The key to success in orthodontic treatments are focused on a good diagnosis, and excellent multi and interdisciplinary communication with other dental specialties, always considering prevention as a primary area. The objective of this study is to determine the prevalence of gingival enlargement and retraction in a patient with orthodontic treatment, in order to know the type of prevalent periodontal alterations in patients with orthodontic appliances.

The sample used is 200 patients who attend orthodontic care at the Hospital Odontológico Monseñor Agustín Hombach of the Universidad Católica de Honduras. This sample was calculated based on a universe of 450 people with a $5 \%$ of sample error, constituting this as a simple random sample. The data collection instrument included: an anamnesis (interrogatories filled in by the patient) and the part of the clinical history (filled by the researcher) in which the enlargement, gingival retraction and bacterial plaque were observed and measured clinically. The results established in the study showed that the prevalence of enlargement was $110(55 \%)$ patients and gingival retraction $21(10 \%)$ patients, the deficiency of oral hygiene is prevalent in patients with orthodontic treatment because of the fixed appliances they use, the risk factors (diet, systemic diseases and medications) do not aggravate the enlargement and gingival retraction in such patients. In the statistical analysis of correlation used, the research hypotheses were verified stating that: there is a correlation between time of the orthodontic treatment and the gingival enlargement in patients with orthodontic treatment, in which there was a positive correlation with a significance rate of 0.021 and that there is correlation of age of the patient with approved gingival retraction with a significance rate of 0.011 .

Keywords: gingival enlargement, gingival retraction, orthodontic treatment, hygiene deficiency. 


\section{INTRODUCCIÓN}

La existencia de alteraciones periodontales y la necesidad del tratamiento ortodóntico se ha evidenciado a lo largo del tiempo, por lo que es necesario entender que pueden llegar a tener mucha relación, para poder solucionar múltiples casos que se presentan en la clínica. Este estudio muestra las condiciones más comunes que pueden llegar a afectar el tratamiento de ortodoncia.

La placa bacteriana es una masa blanda, tenaz y adherente de colonias bacterianas en la superficie de los dientes, la encía, la lengua y otras superficies bucales (incluso las prótesis) (Higashida, 2009), esta está presente en todos los individuos y es capaz de producir alteraciones gingivales, especialmente cuando se combina con la presencia de aparatos ortodónticos, ya que se dificulta la remoción de la placa bacteriana debido a que la aparatología fija en la boca actúa como medio retentivo y complica las técnicas de higiene bucal (Weisson, 2011) formando inflamación y agrandamiento gingival. Por otra parte el movimiento excesivos de las piezas y los efectos de la inflamación del tejido gingival puede llegar a producir un descubrimiento radicular en las piezas dentales.

La importancia es identificar las causantes de estos problemas periodontales, para tratar de ayudar de la mejor manera a los pacientes con tratamiento ortodóntico, que tengan conciencia de lo que puede generar una deficiencia de higiene bucal y para el profesional concientizar al paciente de los problemas que pueden generarse durante el tratamiento de ortodoncia por la prolongada duración del tratamiento (entre18 y 24 meses). (Dra. Erika Truque) El agrandamiento gingival es una alteración que puede llegar a afectar e interrumpir el tratamiento. Ya que impide el movimiento dental, normalmente es autolimitante y responde bien a la limpieza de la placa, al raspado 0 ambos (Soriano, 2014) en cambio, la retracción gingival es un efecto indeseable de la exposición de la superficie radicular. Al igual que el agrandamiento gingival tienen efecto antiestético y puede conducir al desarrollo de sensibilidad dental, una mayor predisposición a la abrasión y a la caries radicular. (Loigge, 2010)

\section{METODOLOGIA}

El enfoque de esta investigación es cuantitativo ya que se usa la recolección de datos y análisis estadísticos para probar hipótesis y analizar resultados (Roberto 
Hernández Sampieri, 2014); Descriptiva, ya que se describen las variables dependientes : agrandamiento y retracción gingival durante el tratamiento ortodóntico; correlacional porque se determinará si, el tiempo del tratamiento y la edad del paciente están correlacionadas con estas alteraciones periodontales en un tratamiento ortodóntico. Es de tipo transversal ya que el estudio se hizo en un periodo de tiempo enero a agosto de 2017. Tiene un diseño no experimental, porque que el agrandamiento y las retracciones gingivales son variables que no son controladas, ya que se necesita conocer si están presentes o no en un tratamiento ortodóntico.

\section{Hipótesis}

- Hi: Si existe prevalencia de la deficiencia de higiene bucal en pacientes con agrandamiento y retracción gingival en pacientes con tratamiento de ortodoncia.

- Hi: Existe correlación de tiempo del tratamiento de ortodoncia con agrandamiento gingival en pacientes con tratamiento de ortodoncia.

- Ho: No existe correlación de tiempo del tratamiento de ortodoncia con agrandamiento gingival en pacientes con tratamiento de ortodoncia.

- Hi: Existe correlación de edad del paciente con retracción gingival.

- Ho: No existe correlación de edad del paciente con retracción gingival.

\section{Metodología}

- Población: La población de la investigación es conformada por pacientes con un tratamiento de ortodoncia que asisten a consulta al Hospital Monseñor Agustín Hombach de la Universidad Católica de Honduras en el año 2017.

- Universo : estimado para realizar el estudio es de 450 pacientes que asisten a atención en la clínica de ortodoncia.

- Muestra : es probabilística ya que los sujetos que se investigaron se tomaron de forma aleatoria al asistir a su consulta en la clínica de ortodoncia por los especialistas de ortodoncia. Mediante el cálculo se obtuvo una muestra de 200 pacientes utilizando un $5 \%$ de error muestral y un universo de 450 pacientes.

\section{Instrumento}

Para poder construir el instrumento de recolección de muestra, se realizó un cuadro de operacionalización de variables, en el cual se conceptualizaron y se operacionalizaron las variables dependientes e independientes asignando indicadores e ítems a cada una de ellas lo cual genero un instrumento de recolección en dos partes: 
- Parte 1 anamnesis (información llenada por el paciente)

- Parte 2 (recopila información del paciente) se mide el agrandamiento y retracción gingival con una sonda periodontal, y control de placa.

Dicho instrumento se aplicó a cada uno de los pacientes con tratamiento de ortodoncia que asisten a su cita en el hospital odontológico Monseñor Agustín Hombach con la especialidad de ortodoncia.

\section{Procedimiento.}

Durante la aplicación del instrumento de la muestra al paciente se le mostró un expediente el cual llenaba preguntas de la anamnesis, seguidamente el investigador procede a llenar la historia clínica, que consiste en medir los agrandamientos y retracciones gingivales con una sonda periodontal y realizar control de placa bacteriana

Al momento de recaudar todos los datos de la muestra se utilizó el programa SPPS para tabular los datos, hacer análisis de frecuencia y tablas contingencia para conocer si una variable depende de la otra. Mediante una prueba de contingencia de" ChiCuadrado" se determinó si se aceptan o rechazan las hipótesis nulas o las hipótesis investigativas.

\section{RESULTADOS}

\section{Gráficos de frecuencia}

Gráfico 1. Frecuencia de tiempo de tratamiento de ortodoncia.

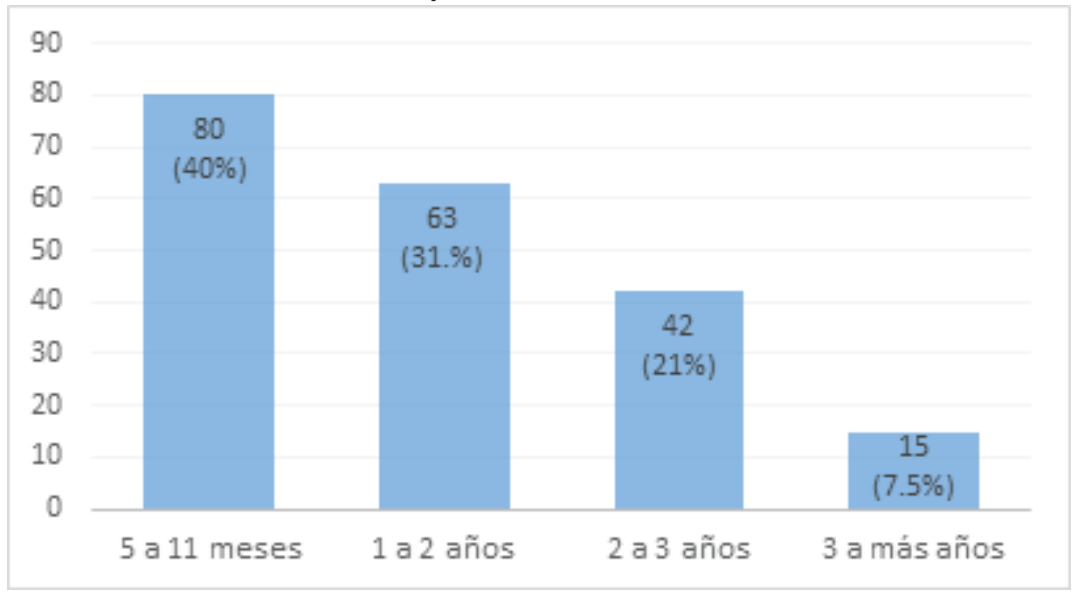


De los 200 pacientes que se les realizó la historia clínica 80 llevaban de 5 a 11 meses con el tratamiento de ortodoncia con un porcentaje de 40\%, 63 tenían de 1 a 2 años con un 31\%, 42 pacientes de 2 a 3 años con $21 \%$ y 15 pacientes con $7.5 \%$ de porcentaje.

Gráfico 2. Frecuencia de cepillado de los dientes.

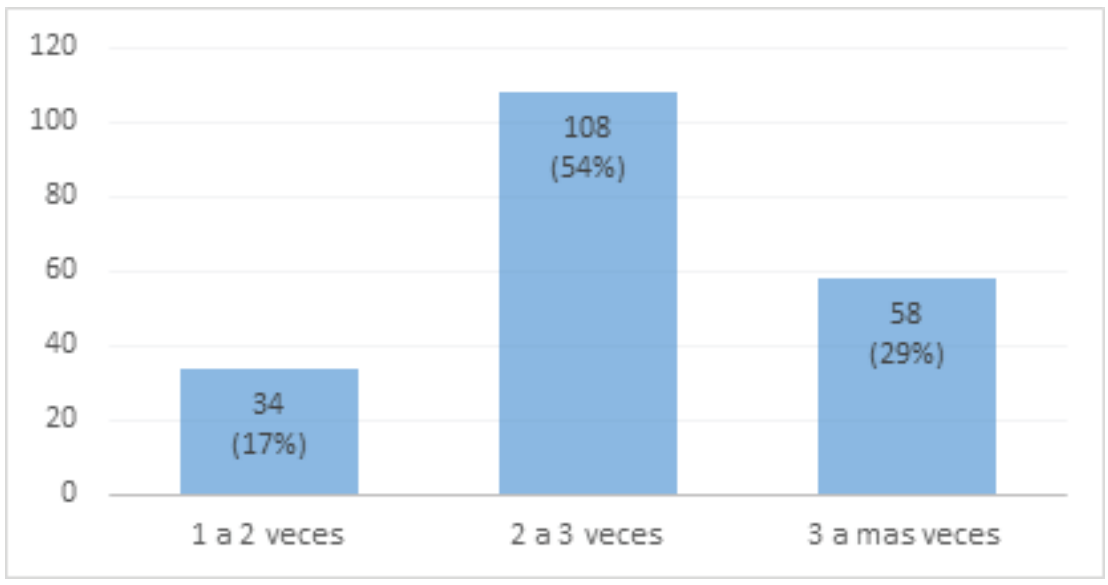

De 200 pacientes que se revisaron para la historia clínica 34 pacientes se cepillan los dientes de 1 a 2 veces con un porcentaje de 17\%, 108 pacientes se cepillan de 2 a 3 veces al día y 58 pacientes con un $29 \%$ de 3 a más veces.

Gráfico 3. Frecuencia de técnica de cepillado.

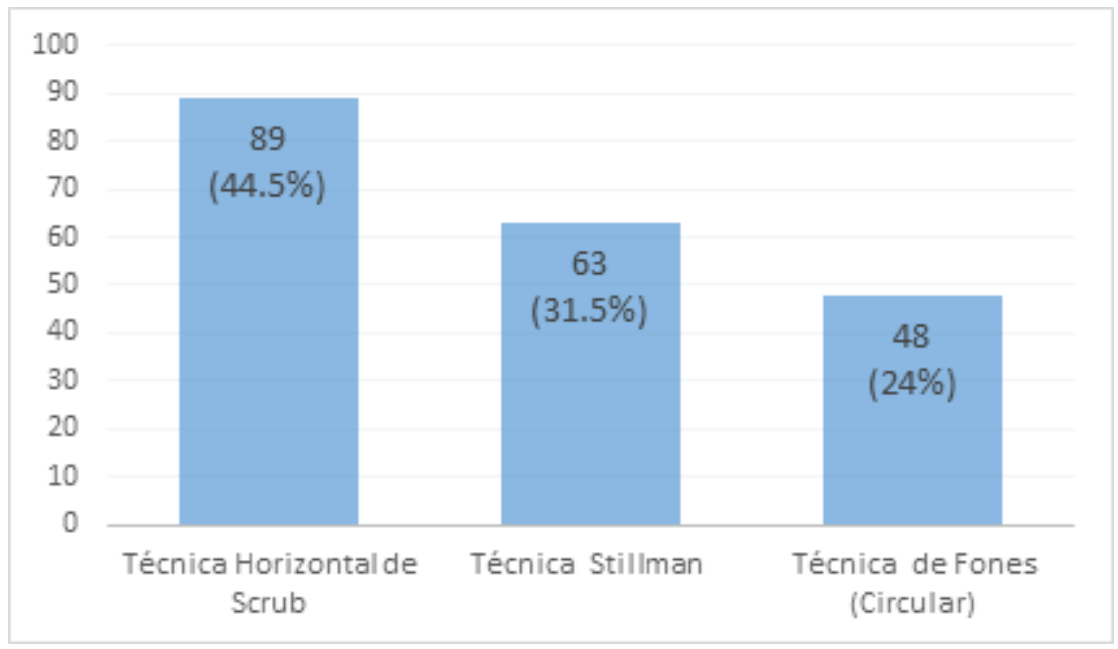


De 200 que se evaluaron 89 con un 44.5\% se cepilla los dientes con una técnica horizontal de Scrub, 63 pacientes con un (31,5\%) hacen su cepillado según la técnica Stillman, 48 pacientes con $24 \%$ de porcentaje se cepillan hacen se cepillado con una técnica de fones (circular).

\section{Gráfico 4. Frecuencia de enfermedad sistémica}

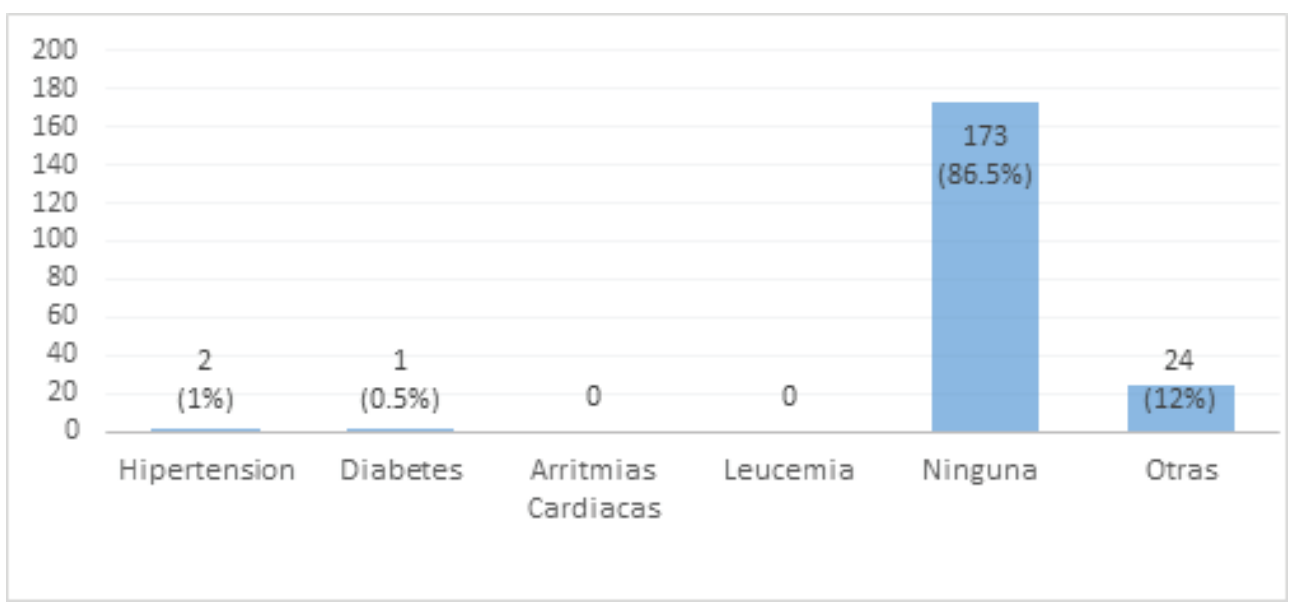

De 200 pacientes que se revisaron 1 paciente padece de diabetes con un $0.5 \%, 2$ pacientes padecen de hipertensión arterial siendo el 1\%, 24 pacientes padecen de otras enfermedades (Rinitis, Gastritis, Convulsiones, Asma, Síndrome de Ovarios Poliquísticos) siendo el $12 \%$ y el $86.5 \%$ de 173 pacientes no padecen de ninguna enfermedad.

\section{Gráfico 5. Frecuencia de consumo de medicamentos}

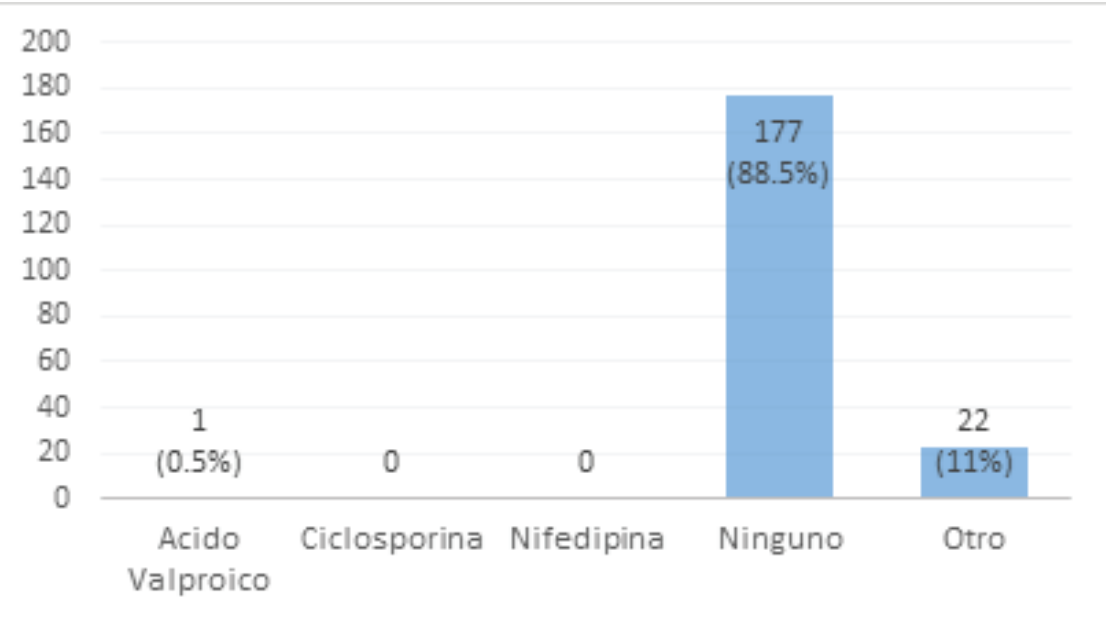


De 200 pacientes que se evaluaron 1 paciente consume ácido valpróico con un 0.5\% de porcentaje, 22 pacientes con un 11\% consumen otros medicamentos (Renitidita, Loratadina, salbutamol), 177 pacientes no consumen ningún medicamento con un $88.5 \%$ de porcentaje.

Gráfico 6. Frecuencia de índice de placa bacteriana.

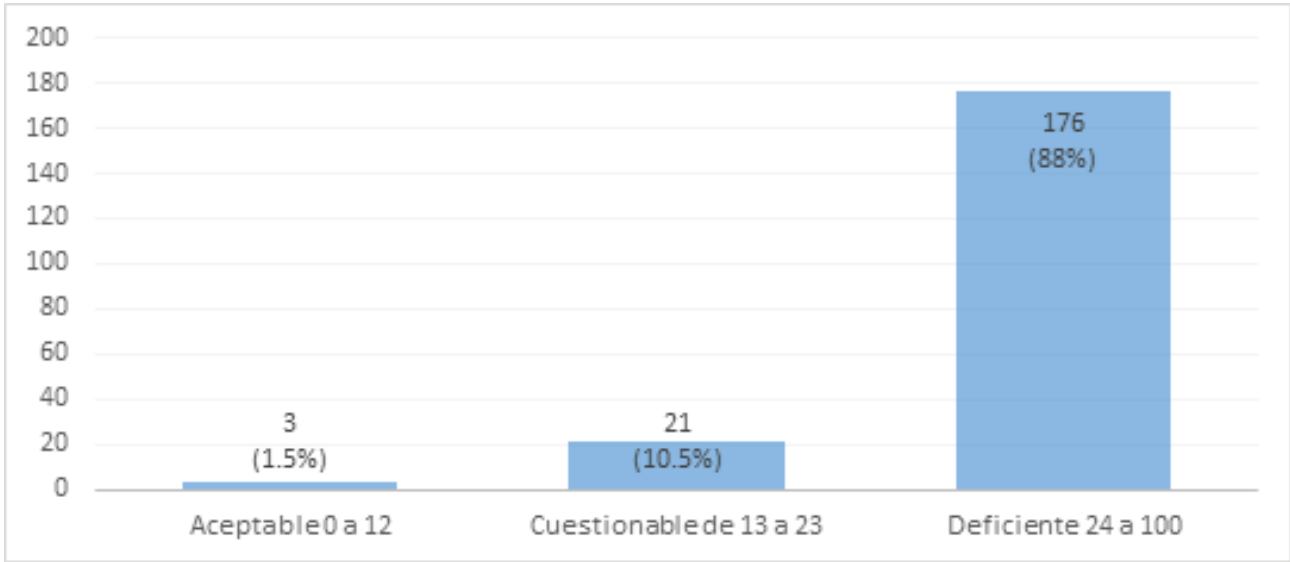

Los pacientes evaluados de una muestra de 200 el 1.5\% de 3 pacientes el índice de placa es aceptable 0 a 12\%, 21 pacientes con un 10.5\% presentan un índice de place cuestionable de 13 a 23\%, 176 pacientes con un $88 \%$ es deficiente 24 a $100 \%$.

Gráfico 7. Frecuencia de prevalencia de agrandamiento y retracción gingival.

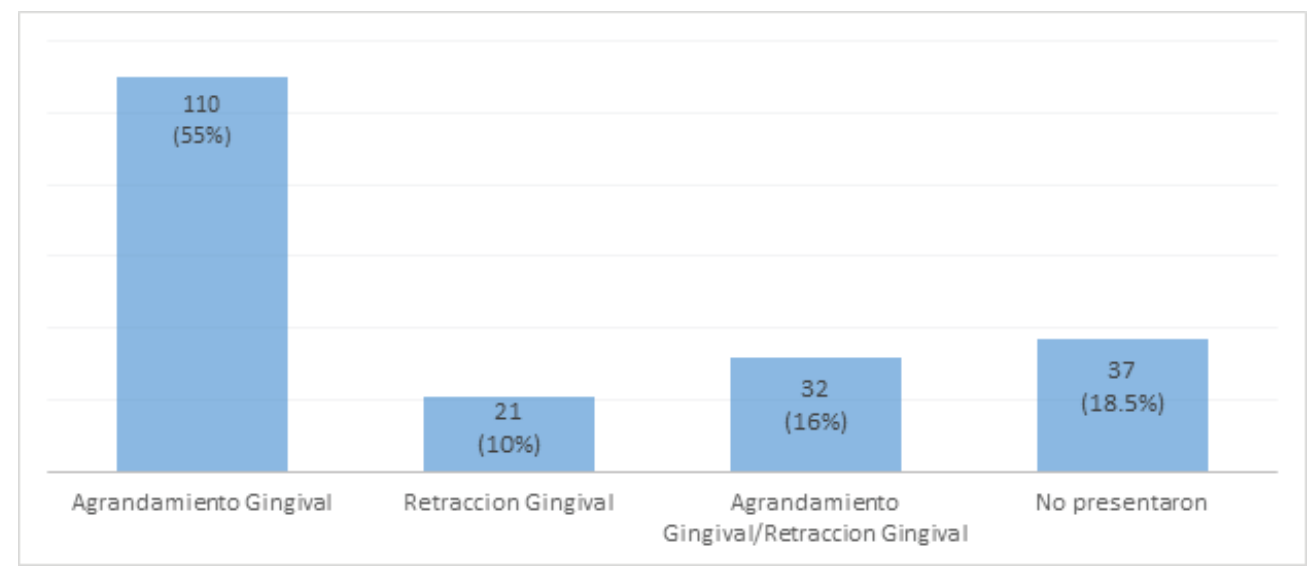


De 200 pacientes que se evaluaron 110 pacientes presentan agrandamiento gingival con un 55\%, 21 pacientes con un 10\% presentaron retracción gingival, 32 pacientes presentaron ambas alteraciones periodontales durante, 37 pacientes con un $18.5 \%$ no presentaron agrandamiento ni retracción gingival durante el tratamiento de ortodoncia.

\section{Tablas correlacionales}

Tabla 1. Tiempo de tratamiento de ortodoncia-prevalencias de agrandamiento gingival.

\begin{tabular}{|l|r|}
\hline \multicolumn{2}{|c|}{ pruebas de chi-cuadrado } \\
\hline & $\begin{array}{c}\text { significación asintótica } \\
\text { (bilateral) }\end{array}$ \\
\hline chi-cuadrado de Pearson & .021 \\
\hline
\end{tabular}

Mediante el uso del instrumento estadístico de chi-cuadrado se realizó la comparación del Tiempo de Ortodoncia con la Prevalencia de Agrandamiento Gingival con un índice de significancia de de 0.21, por lo tanto, se acepta la Hipótesis Investigativa que existe una correlación del tiempo del tratamiento de ortodoncia con el agrandamiento gingival en pacientes con un tratamiento de ortodoncia, rechazando la Hipótesis Nula que no existe una correlación del tiempo del tratamiento de ortodoncia con el agrandamiento gingival en pacientes con un tratamiento de ortodoncia.

\section{Tabla 2. Edad - prevalencia de retracción gingival.}

\begin{tabular}{|l|c|}
\hline \multicolumn{2}{|c|}{ Prueba de Chi-Cuadrado } \\
\hline & $\begin{array}{c}\text { Significación asintótica } \\
\text { (bilateral) }\end{array}$ \\
\hline Chi-cuadrado de Pearson & .011 \\
\hline
\end{tabular}

En base al instrumento estadísticos de Chi-Cuadrado se realizó la comparación de Edad con Prevalencia de Retracción Gingival índice de significancia de 0.011, por lo que se acepta la Hipótesis de Investigación que hay una correlación de edad del paciente con retracción gingival con tratamiento de ortodoncia, donde se rechaza la Hipótesis Nula que no hay una correlación de edad de paciente con retracción gingival en pacientes con tratamiento de ortodoncia. 


\section{DISCUSIÓN}

Según un estudio realizado en la Universidad Católica de Santiago de Guayaquil por el Dr. Roberto C. Lendergerber W. Los resultados que obtuvieron de prevalencia de agrandamiento gingival fue de 43 pacientes (36\%) y retracciones gingivales 2 pacientes (2\%) (Weisson, 2011) , al igual que nuestro estudio 110 (55\%) pacientes presentaron agrandamiento gingival y $21(10 \%)$ presentaron retracción gingival, siendo el agrandamiento gingival con mayor prevalencia.

Durante el tratamiento de ortodoncia se dificulta la remoción de placa bacteriana debido a la aparatología fija en la boca, ya que actúa como un medio retentivo y complica las técnicas de higiene y no va a permitir el fácil acceso de las cerdas del cepillo. Los resultados encontrados en los pacientes fue hasta $87.14 \%$ (Weisson, 2011) En cambio el estudio realizado, los resultados fueron que 176 (88\%) pacientes tiene una deficiencia de higiene bucal. Comprobando la teoría establecida del artículo. Según el estudio realizado por el Dr. Roberto C. Ledergerber W. Se observó que ninguno de los pacientes ha referido en las historias clínicas alguna enfermedad sistémica, problemas hormonales o el consumo de medicamentos que pudiera estar relacionado con agrandamiento gingival (Weisson, 2011), siendo comprobado también en este estudio que dichos factores de riesgo no agravan ni están relacionados con agrandamiento ni retracciones gingivales.

Mediante la prueba de "Chi cuadrado" se realizaron pruebas correlacionales, en las cuales se aceptó las hipótesis investigativas: "Existe una correlación de tiempo de tratamiento y agrandamiento gingival en pacientes con tratamiento de ortodoncia", Por el tiempo prolongado que utiliza el paciente la aparatología fija. "Existe una correlación de edad del paciente con retracciones gingivales en pacientes con un tratamiento de ortodoncia", En este caso es ya que cierto grupo de pacientes presentan esta alteración.

\section{CONCLUSIONES}

- Se concluyó que el agrandamiento gingival es una de las alteraciones que más prevalece en el tratamiento de ortodoncia correspondiendo a un incide de $55 \%$ de la población y siendo menos prevalente la retracción en un 10\%.

- Según los resultados obtenidos la deficiencia de higiene bucal es un factor preva- 
lente durante el tratamiento de ortodoncia, ya que la aparatología fija que utiliza el paciente durante el tratamiento retiene placa dentobacteriana, impidiendo una buena higiene bucal el paciente. Siendo el porcentaje promedio de la investigación un $88 \%$ el cual se considera un índice elevado.

- Se determinó que los factores de riesgo durante el tratamiento (medicamentos, enfermedades sistémicas y dieta) no agravan el agrandamiento y retracción gingival ya que los pacientes se encuentran con un buen estado de salud y en su dieta no consumen muchos alimentos ácidos.

- Los resultados reflejaron que el tiempo de tratamiento está correlacionado con la presencia de agrandamiento gingival por el tiempo prolongado que tiene el tratamiento, en cambio la retracción gingival esta correlacionada con la edad del paciente ya que cierto grupo presenta esta enfermedad, la edad que presenta mayor retracción es en pacientes de 23 años.

\section{AGRADECIMIENTOS}

Muchas gracias a la Dra. Lourdes Fernández por asesorarme en la parte metodológica e impulsar a realizar este proyecto y poder desarrollarlo, a la Dra. Hortencia por asesorarme en la parte técnica durante todo el proceso.

\section{REFERENCIAS BIBLIOGRÁFÍCAS}

Dra. Erika Truque, D. B. (n.d.). Hiperplasia Gingival y Tratamiento Ortodóncico : Caso Clínico . iDental , 33-46.

Higashida, B. Y. (2009). Odontología Preventiva (Segunda Edición ed.). (N. L. Carbajal, Ed.) Ciudad de Mexico, Mexico: McGraw Hill.

Loigge, O. G. (2010). Recesiones Gingivales y Tratamiento de Ortodoncia. Argentina.

Roberto Hernández Sampieri, C. F. (2014). Metodología de la Investigación . In C. F. Roberto Hernández Sampieri, Metodología de la Investigación (6ta Edición ed., p. 600). Ciudad de México, D.F, México: McGraw-Hill/Interamericana Editores, S.A DE C.V.

Soriano, R. G. (2014). Hiperplasia gingival en paciente con tratamiento ortodóntico. Guayaquil.

Weisson, R. C. (2011). Problemas Periodontales más frecuentes relacionados al Tratamiento Ortodóntico en pacientes de la Clínica de Posgrado de la Universidad Católica de Santiago de Guayaquil. MedPre , 1, 69-73. 